Meta

Journal des traducteurs

Translators' Journal

\title{
Localisation, traduction et diversité sociolinguistique en Afrique sub-saharienne : stratégies et perspectives
}

\section{Jeannine Gerbault}

Volume 55, numéro 4, décembre 2010

De la localisation à la délocalisation - le facteur local en traduction From Localization to Delocalization - The Local Factor in Translation

URI : https://id.erudit.org/iderudit/045693ar

DOI : https://doi.org/10.7202/045693ar

Aller au sommaire du numéro

Éditeur(s)

Les Presses de l'Université de Montréal

ISSN

0026-0452 (imprimé)

1492-1421 (numérique)

Découvrir la revue

Citer cet article

Gerbault, J. (2010). Localisation, traduction et diversité sociolinguistique en Afrique sub-saharienne : stratégies et perspectives. Meta, 55(4), 817-844. https://doi.org/10.7202/045693ar
Résumé de l'article

Pour les langues d'Afrique sub-saharienne, localisation et traduction interviennent dans un double jeu de contraintes linguistiques et sociolinguistiques et font face à deux tendances parfois conflictuelles : la mondialisation, d'une part, et les politiques déclarées de promotion de la diversité linguistique, d'autre part. En nous focalisant sur les pratiques s'appuyant sur les technologies de l'information, à la fois outils de la localisation et de la traduction en langues africaines et moyens de développement de celles-ci, nous présenterons les problèmes liés à ces activités et exposerons comment ces activités s'organisent et se déploient dans le contexte actuel. Nous ferons état de la présence des langues africaines sub-sahariennes dans les environnements numériques et proposerons une analyse de la dynamique de la traduction où s'articulent des processus et des pratiques qui agissent selon deux axes, l'un de facilitation et l'autre de complexification.
Ce document est protégé par la loi sur le droit d'auteur. L’utilisation des services d’Érudit (y compris la reproduction) est assujettie à sa politique d'utilisation que vous pouvez consulter en ligne.

https://apropos.erudit.org/fr/usagers/politique-dutilisation/ 


\title{
Localisation, traduction et diversité sociolinguistique en Afrique sub-saharienne: stratégies et perspectives
}

\author{
JEANNINE GERBAULT \\ Université de Bordeaux, Bordeaux, France \\ jannine.gerbault@u-bordeaux3.fr
}

\begin{abstract}
RÉSUMÉ
Pour les langues d'Afrique sub-saharienne, localisation et traduction interviennent dans un double jeu de contraintes linguistiques et sociolinguistiques et font face à deux tendances parfois conflictuelles: la mondialisation, d'une part, et les politiques déclarées de promotion de la diversité linguistique, d'autre part. En nous focalisant sur les pratiques s'appuyant sur les technologies de l'information, à la fois outils de la localisation et de la traduction en langues africaines et moyens de développement de celles-ci, nous présenterons les problèmes liés à ces activités et exposerons comment ces activités s'organisent et se déploient dans le contexte actuel. Nous ferons état de la présence des langues africaines sub-sahariennes dans les environnements numériques et proposerons une analyse de la dynamique de la traduction où s'articulent des processus et des pratiques qui agissent selon deux axes, l'un de facilitation et l'autre de complexification.
\end{abstract}

\begin{abstract}
Localization and translation for languages of sub-Saharan Africa are done within a double set of linguistic and sociolinguistic constraints, and they face two often conflicting trends, on the one hand globalization, and on the other hand the declared policies of promotion of language diversity. I will focus on the area of information technologies, which are both the tools for localization and translation in African languages and the means for developing them. I will present the problems encountered in translation and localization activities, and how these activities develop in the present context. I will review the presence of African languages in digital environments and propose an analysis of the dynamics of translation in which processes and practices interact along two axes, facilitation and complexification.
\end{abstract}

\section{MOTS-CLÉS/KEYWORDS}

Afrique sub-saharienne, localisation, ingénierie linguistique, langues et développement sub-Saharan Africa, localization, language engineering, languages and development

Chercher l'homogénéité, c'est aller à l'encontre de la vie: en biologie, le développement des êtres vivants passe par la complexification des cellules et de leur assemblage.

(Adams 2004)

Parmi les effets des changements politiques, sociaux et technologiques qui ont marqué la fin $\mathrm{du} \mathrm{xx}^{\mathrm{e}}$ siècle et le début du $\mathrm{xxI}^{\mathrm{e}}$ siècle, l'augmentation considérable du volume de la communication interlinguistique et le développement des technologies de l'information et de la communication représentent un double défi dans le domaine de la traduction et de la localisation. En effet, l'étude de leurs problématiques ne peut 
aujourd'hui faire d'impasse géographique, et le développement des technologies de l'information et de leurs applications est à la fois un outil de développement de la traduction et un révélateur des pratiques.

Les sociétés humaines sont multilingues, et la mondialisation appelle à l'utilisation et à l'acquisition accrues de plusieurs langues pour la communication, médiatisée ou non par les technologies de l'information. Les activités de communication sont soumises à un ensemble de contraintes, dont le volet linguistique est l'un des éléments, et on fait effectivement de plus en plus de lecture, d'analyse et de traduction dans des langues de plus en plus nombreuses. Dans le cadre de l'Union européenne, la complexité des problèmes à gérer pour l'intercompréhension et la mise à disposition de traductions, d'outils de traduction et de contenus localisés est souvent évoquée (notamment dans le cadre de l'Observatoire européen du plurilinguisme ${ }^{2}$; voir aussi, par exemple, le récent colloque de Strasbourg ${ }^{3}$ ), mais on connaît moins les problèmes qui se posent sur le continent africain. Ils se présentent dans cette région du monde enracinés dans un ensemble spécifique encore plus complexe de contingences et d'exigences parfois contradictoires.

Nous aborderons la problématique de la localisation et de la traduction en Afrique en limitant notre perspective à l'Afrique sub-saharienne. Les pays arabophones du nord du continent et l'Afrique du Sud ne seront mentionnés que pour établir des comparaisons et lorsque problèmes et solutions se recoupent à travers ces régions. Les raisons de ce choix apparaîtront au cours de notre propos.

Nous mettrons en avant tout d'abord le double jeu de contraintes linguistiques et sociolinguistiques dans lequel interviennent traduction et localisation en Afrique sub-saharienne et les problèmes qu'elles posent. Ces contraintes sont à l'œuvre face à deux tendances parfois conflictuelles, qui portent en elles dans une large mesure les besoins en traduction: d'une part, la mondialisation - multiplication des échanges et mobilité des personnes et des biens (exigeant, par exemple, la localisation de sites commerciaux) -, et d'autre part, les politiques officielles de promotion de la diversité linguistique (UNESCO, Francophonie institutionnelle, par exemple).

Nous exposerons comment les activités de traduction et de localisation s'organisent et se déploient dans le contexte actuel, en nous focalisant sur les pratiques s'appuyant sur les nouvelles technologies de l'information. En effet, leur utilisation n'est plus considérée aujourd'hui comme un simple ajout aux techniques de communication plus traditionnelles, mais au contraire les outils du numérique, et surtout la Toile et la téléphonie mobile, sont devenus partout des éléments structurants pour l'organisation de la communication, et reflètent donc les processus, stratégies et tendances à l'œuvre dans le domaine de la traduction.

L'enjeu de la traduction et de la localisation sur la Toile et, plus largement, pour l'utilisation des outils nés de la technologie numérique, est majeur. En effet, comme l'écrivait déjà Guédon (1996), on assiste à un transfert massif de toutes les cultures du monde dans les systèmes numériques, si bien que dans 20 ou 30 ans au plus, tout ce qui ne sera pas codable en 0 et 1 sera complètement marginalisé au point d'être oublié, comme les manuscrits médiévaux. 


\section{Le contexte}

\subsection{Le contexte linguistique}

Le continent africain abrite un tiers des langues du monde et c'est dans les pays d'Afrique sub-saharienne que l'on en trouve l'une des plus hautes densités ${ }^{4}$, certaines ayant relativement peu de locuteurs. Ces pays se caractérisent ainsi à la fois par une grande diversité linguistique et par des rapports de force inégalitaires entre langues européennes et langues africaines, rapports issus de l'histoire coloniale. Nous sommes donc là sur un terrain d'étude et d'action à la fois riche et complexe pour la traduction et la localisation.

Pour des raisons liées au contexte historico-politique, les langues africaines endogènes, dont les découpages politiques ont fait que certaines d'entre elles sont transfrontalières, coexistent avec les langues de grande communication (LGC) ou «géolangues» que sont l'anglais, le français et le portugais (l'espagnol est présent à un moindre degré). Ces langues africaines ont aujourd'hui pratiquement toutes été décrites sur les plans phonologique, morphologique et syntaxique, mais elles ne sont pas toutes standardisées et beaucoup sont restées des langues à tradition orale (et donc à corpus oral) et à usage social oral. Pour celles-ci, le développement terminologique est resté le plus souvent limité aux domaines traditionnels et informels d'activité.

\subsection{Le contexte social et économique}

Sur cet arrière-plan linguistique, la problématique abordée ici est à considérer dans son contexte social et économique. Plus de $40 \%$ de la population adulte d'Afrique est analphabète (estimation de l'UNESCO [2008a] ; monde: $25 \%$ ). Ceci est un paramètre important lorsqu'on sait que l'écrit est encore largement dominant dans la communication médiée par les technologies de l'information. L'un des corollaires du fait que de nombreuses langues sont restées essentiellement à usage oral est que, parmi la population lettrée, beaucoup de personnes ne maîtrisent pas l'écrit dans leur propre langue.

Pourtant, il est vrai que, partout dans le monde, y compris dans les pays du Sud et en Afrique sub-saharienne, de nouvelles pratiques de communication se sont implantées. Les possibilités de communiquer à distance se sont vues multipliées: la téléphonie mobile, qui permet d'utiliser les langues à l'oral, occupe de plus en plus de terrain. Avec les textos (SMS), le courriel, les forums, les blogues, les messageries instantanées sur Internet (Skype ou autres), on peut - et les diasporas, notamment, ne manquent pas d'en profiter - communiquer dans sa langue d'origine par écrit avec ou sans la voix, et le monde de l'écrit devient plus abordable pour les scripteurs à compétences restreintes. Nous voyons donc là un potentiel d'extension de la communication dans toutes les langues, et de nouveaux espaces pour la traduction et la localisation. Et il est vrai qu'à mesure que la technologie est apprivoisée, la créativité individuelle se manifeste: par exemple, les lecteurs deviennent journalistes (les publications du type Gazette du Net se multiplient) et des services d'aide à la traduction sont proposés par des volontaires.

Mais à côté de cela, même si les gouvernements se sont engagés dans des programmes de développement de leurs équipements de communication, si les personnes lettrées s'approprient progressivement le cyberespace, si l'accès à la Toile gagne du 
terrain partout et si les nouvelles pratiques sont associées à de nouveaux modes de coexistence des langues, si, enfin, Internet est en train de devenir un média bien différent du mass media traditionnel parce que ses contenus sont élaborés par un grand nombre de personnes, et non plus par un petit nombre de spécialistes, les problèmes d'ordre économique et social ne peuvent être occultés lorsqu'on aborde la problématique de la traduction et de la localisation sur la Toile et pour les autres usages issus de la technologie numérique. En effet, on peut s'interroger sur la pertinence des technologies de l'information dans un continent où les besoins vitaux eau, nourriture, santé - continuent de poser massivement problème.

Pour répondre, nous reprenons d'abord les arguments d'Acacia, programme canadien du Centre de recherches pour le développement international (CRDI), qui vise à déterminer le potentiel qu'offrent les technologies de l'information et de la communication (TIC) pour rendre autonomes les communautés pauvres d'Afrique: les TIC ne sont pas un luxe; elles sont transformatives sur plusieurs plans, car elles ont la capacité de faciliter le fonctionnement des services et commerces traditionnels, elles sont un secteur économique à part entière, et elles permettent la création de modèles économiques originaux et de nouveaux types d'organisation sociale. Nous reviendrons sur les enjeux de l'utilisation des technologies de l'information dans les sociétés africaines dans la section suivante.

\subsection{Le contexte sociolinguistique}

Néanmoins, s'agissant de l'utilisation des langues africaines, un état de fait perdure: ce sont les LGC qui sont les langues dominantes et qui sont utilisées très majoritairement dans les fonctions de communication valorisantes. Comme le rappelait encore le rapport général de la conférence régionale "Politiques nationales: le rôle des langues transfrontalières et la place des langues de moindre diffusion en Afrique centrale» tenue à Yaoundé, Cameroun, en octobre 2007,

[1] a marginalisation des langues africaines, une des conséquences de la colonisation, a conduit à l'intériorisation, au sein des populations, d'un sentiment d'infériorité vis-àvis des langues héritées de la colonisation qu'il fallait maîtriser pour ne pas être en marge des prises de décision et de l'exercice du pouvoir. (Conférence régionale sur les Politiques nationales: le rôle des langues transfrontalières et la place des langues de moindre diffusion en Afrique centrale, Rapport Général 2007: 46)

Malgré les politiques déclarées de diversité linguistique, le multilinguisme africain est donc loin d'être socialement "équilibré», et malgré le rôle des outils des technologies de l'information pour la communication personnelle et professionnelle et leur potentiel pour la vitalité des langues, la connaissance d'une LGC continue d'être pratiquement toujours supposée lorsqu'on utilise la technologie numérique. Or, la plupart des locuteurs de langues africaines n'ont pas les compétences langagières nécessaires pour accéder aux technologies de l'information et de la communication dans des langues comme l'anglais ou le français.

Il faut ajouter que les scripteurs africains, comme tous les scripteurs, écrivent pour être lus, et recourent aux LGC pour avoir le plus large lectorat possible. Le moindre prestige qui est attaché aux langues africaines a été longtemps, et demeure encore pour beaucoup, un élément de non-motivation pour l'apprentissage et la pratique de la lecture et de l'écriture dans ces langues. 
Par conséquent, si les wikis, blogues et forums occupés par le grand public sont autant d'espaces qui illustrent le droit aujourd'hui partagé de s'exprimer et d'être fournisseur d'information, ils n'échappent pas aux conflits entre les motivations individuelles des utilisateurs dans cette région du monde: c'est-à-dire qu'on y trouve surtout des contenus francophones ou anglophones, parce que les internautes écrivent pour être lus et compris par le plus grand nombre, mais que certains Africains cherchent aussi à localiser et à traduire pour exprimer les identités nationales ou régionales.

\section{Traduction et localisation}

\subsection{Les enjeux et les besoins}

Osborn (2010) traite en détail des enjeux de la localisation, et reprend les arguments avancés par diverses organisations et associations depuis le début de la mondialisation des échanges et la pénétration d'Internet sur tous les continents: la localisation est essentielle si l'on veut que les populations des pays les plus pauvres aient un meilleur accès aux technologies de l'information et de la communication, que ces technologies correspondent davantage aux vies, aux besoins et aux aspirations de ces populations et, en bout de ligne, que soit réduite la fracture numérique. Il met en avant l'importance de la localisation dans le contexte africain pour le développement et l'éducation à long terme, et affirme qu'il y a davantage dans la localisation qu'il n'y paraît de prime abord; et que ceci est vrai spécialement dans cet environnement complexe africain caractérisé par le multilinguisme, une faible standardisation des langues, des politiques linguistiques fluctuantes, des langues transfrontalières, un accès aux technologies de l'information très inégalement distribué. Pour cet auteur, que nous rejoignons sur ce point, non seulement les aspects techniques et organisationnels de la localisation sont affectés par ces facteurs, mais la localisation a le potentiel d'influer en retour sur ces facteurs. La localisation est en fait un élément clé pour combler la fracture numérique et les autres fractures "analogues», et augmenter la pertinence des technologies de l'information.

Les besoins en traduction sont énormes. On a compris que le multilinguisme et le plurilinguisme ne sont pas des phénomènes restreints en Afrique sub-saharienne. Selon Diki-Kidiri:

Dans plusieurs pays d'Afrique, le français a aujourd'hui le statut de langue officielle ou co-officielle. De plus, sur le plan sociologique, il occupe souvent une place si importante que personne ne pense à le remplacer, mais malgré cela, on ne peut pas se passer des langues africaines quand on veut vraiment œuvrer au développement des populations de nos pays.

En effet, même si les cadres et les techniciens de la santé, de la culture, de l'industrie, etc. sont formés en français dans des écoles francophones, dans leurs activités professionnelles quotidiennes, ils ont à côtoyer des gens qui ne connaissent pas le français. Par exemple, les vétérinaires qui travaillent dans le milieu des éleveurs ou les techniciens affectés au développement des milieux ruraux doivent transmettre leurs connaissances à des gens qui ne parlent pas le français.

Malheureusement, ces spécialistes n'ont pas appris à exprimer leur savoir dans les langues africaines. Il faut donc les y aider en rendant les langues africaines capables de supporter et de véhiculer les connaissances modernes. (Diki-Kidiri 2004: 65) 
Les besoins sont énormes et permanents dans d'autres domaines aussi, comme ceux de la justice et de la santé, par exemple.

On se rappellera que, dès le début de la pénétration européenne en Afrique subsaharienne, les membres des différents groupes religieux ont commencé à traduire vers les langues africaines pour leurs activités d'évangélisation. La traduction des textes religieux en Afrique a donc une longue histoire, qui se poursuit aujourd'hui, et ces textes constituent d'ailleurs les premiers travaux de traduction réalisés pour les langues de cette région. On trouvera un intéressant historique de la traduction de la Bible en Afrique dans le texte de Kenmogne, directeur de la Cameroon Association for Bible Translation and Literacy, avec cette formulation ad hoc de la nécessité de localiser en traduisant:

Les Africains ne peuvent logiquement que rencontrer des difficultés à déchiffrer ce message [celui de la Bible] dont la formulation n'avait pas pris en compte leur contexte ethnolinguistique particulier. Dans ces conditions, le message biblique peut légitimement devenir une source de confusion chez l'Africain. Alors surgit l'urgence de la traduction de la Bible comprise ici comme formulation du message divin dans une langue qui parle à l'Africain en utilisant les formes de communication qui sont socialement et culturellement appropriées. (Kenmogne 2002:1)

Toutefois, et bien que les traductions des textes religieux aient été réalisées par les linguistes et missionnaires au sein de la Société internationale de linguistique (SIL), les exigences en matière d'harmonisation et de cohérence des pratiques étaient bien différentes pour les textes religieux de ce qu'elles sont aujourd'hui dans un monde globalisé et informatisé.

Par ailleurs, et ici encore hors de toute référence aux technologies de l'information, Tervonen (2003) pose le problème de la diffusion de la littérature africaine. Certes, les auteurs africains écrivent très majoritairement en anglais ou en français, et non dans leurs langues d'origine (nous avons vu pourquoi), mais la littérature africaine reste peu traduite, et leurs textes ne sont pas accessibles dans plusieurs langues.

Sans traductions, la littérature africaine peut-elle prétendre à être une «littérature mondiale»? [...] [1]a traduction reste chère pour l'éditeur qui doit s'acquitter de l'achat des droits et des honoraires du traducteur, tout en assumant le risque financier que représente toute publication d'auteurs méconnus, qui plus est africains. [...] La recherche sur la traduction s'est peu intéressée à la littérature africaine. La traductologie, longtemps marquée par des schémas simplistes et binaires «fidélité-trahison», "ciblistesourcier", s'accommodait difficilement de l'étude de textes africains, caractérisés par une hybridité et une multiplicité de langues. [...] Il est étonnant de constater que sur un continent où la pluralité des langues est une constante de la vie quotidienne, où les pidgins et les parlers urbains se développent, où l'interprète a joué un rôle capital pendant l'époque coloniale, où une des caractéristiques fondamentales des littératures est leur conscience linguistique - bref, sur un continent où la traduction est omniprésente -, elle soit si peu étudiée. (Tervonen 2003)

Les rencontres internationales et interafricaines se succèdent aujourd'hui pour répondre aux besoins de production de contenus traduits ou localisés en langues africaines. Citons d'abord le «Forum international de Bamako sur le multilinguisme», qui s'est tenu en janvier 2009: Bekele, du Bureau Régional Africain de l'Internet Society (ISOC), y faisait état du consensus international relatif aux bénéfices en termes 
d'harmonie sociale et de développement économique de la reconnaissance du multilinguisme et de l'acceptation de la diversité, et des besoins en matière de traduction et d'interprétation pour les nombreuses langues africaines, de manière à ce qu'elles soient préservées et présentes sur Internet.

Lors de la récente Conférence internationale de Yaoundé «Diversité culturelle et Internet multilingue en Afrique», tenue en décembre 2009, les questions essentielles à propos de l'accès à Internet dans un contexte multilingue et multiculturel portaient sur les attentes du continent africain d'un Internet dont environ $80 \% \mathrm{du}$ contenu est en anglais, sur la nature de sa contribution au développement de l'Afrique, et sur les moyens d'éviter qu'Internet ne contribue à la diffusion d'un modèle monoculturel. Les recommandations visaient évidemment des «solutions africaines pour des problèmes africains», c'est-à-dire l'obligation pour Internet de "parler les langues africaines».

Enfin, à côté de ces besoins de traduction pour mise à disposition de contenus avec ou sans les outils de communication les plus modernes aux fins de rayonnement des cultures africaines, et d'accès aux moyens de communication dans ces langues, les besoins de traduction et de localisation de contenus pour l'enseignement en langues nationales ou régionales dans les systèmes éducatifs ont été exprimés à plusieurs reprises depuis quelques décennies dans les différents pays d'Afrique sub-saharienne. Les réponses à ces besoins ont été diversement construites, souvent sans grande conviction et sans grand investissement de la part des gouvernements des pays concernés, en raison des relations de pouvoir et de domination entre langues ${ }^{5}$.

Il est intéressant de noter que lors de sa première édition en 2006, le colloque "eLearning Africa ${ }^{6}$ » avait mis en bonne place sur son agenda les questions relatives aux langues africaines et aux besoins de traduction:

Pour que l'elearning [sic] ait un réel impact en Afrique, il faut y soutenir une industrie du contenu éducatif parfaitement adapté, qui respecte et prenne en compte les besoins de diversité culturelle, linguistique et structurelle propre à ce continent.

Dotée de quelque 2000 langues parlées, ce qui représente un tiers des langues parlées dans le monde, l'Afrique ne peut ignorer la question de la diversité des langues et des cultures quand il s'agit de construire et développer une Société d'Information et du savoir. De nombreux outils libres - open source -, plateformes et stratégies de gestion de contenus y seront présentés et discutés, permettant une utilisation optimale en terme de localisation et d'adaptation de contenus importés.

Cette thématique a été régulièrement absente dans les éditions suivantes. Nous voyons là un constat de prise de conscience de la complexité et de la lourdeur du travail à réaliser pour traduire et localiser.

Qu'il s'agisse de rayonnement culturel, d'ouverture sur le monde, ou de formation initiale ou continue, les tâches et problèmes linguistiques et technologiques liés à la traduction sont lourds à gérer dans cette région sub-saharienne, et nous allons maintenant les aborder.

\subsection{Les aspects linguistiques de la traduction}

\subsubsection{Standardisation et création terminologique}

Comme nous l'avons dit, pour un grand nombre de langues d'Afrique sub-saharienne, le système orthographique est encore à stabiliser. Selon Aito: 
Un nombre important de langues sur le continent sont à tradition orale et connaissent des conventions d'écriture fluctuantes, malgré l'adoption plus récente de l'alphabet arabe ou latin, et du protocole API pour déceler phonétiquement les sons. La segmentation des unités lexicales mérite d'être stabilisée, et les temps verbaux ${ }^{7}$ entraînent souvent des formules périphrastiques sans blancs typographiques. Les marques du nombre sont difficilement perceptibles et ne sont pas unanimes. Tout cela pour dire qu'il y a du travail sur la codification de plusieurs des langues dites minoritaires in situ. (Aito 2005: 15)

Le travail à réaliser pour la création de vocabulaire et la diffusion terminologique dans les langues d'Afrique sub-saharienne est énorme. En effet, l'une des conséquences du statut de ces langues - en coexistence avec des LGC et minorisées - est que les termes nécessaires à la communication dans certains secteurs d'activité sont absents de leur vocabulaire ou, au mieux, inconnus de leurs locuteurs. De plus, même parmi les langues africaines, la tendance, lorsqu'on traduit, consiste souvent, comme partout, à utiliser quelques langues numériquement dominantes. Il ne s'agit pas seulement des termes du domaine des technologies de l'information, mais de tout un ensemble de termes de divers domaines d'activité. Depuis plusieurs années pourtant, la traduction dans des domaines spécialisés - lexique de la santé, de l'élevage, des termes juridiques, des mathématiques, etc. - a été entreprise par des linguistes des pays concernés, d'abord au sein de l'ACCT (Agence de coopération culturelle et technique), puis au sein du Réseau international francophone d'aménagement linguistique $(\mathrm{RIFAL})^{8}$. Et concernant les termes relatifs aux technologies numériques récentes, Gaudin écrit:

[1]'enjeu est de rendre disponibles les termes nécessités par la diffusion de connaissances ou de technologies nouvelles. Former en sängö, en ouolof, ou en bambara de nouveaux utilisateurs, ni francophones ni anglophones, à l'utilisation d'un logiciel suppose que le formateur parvienne à exprimer dans ces langues les connaissances techniques qu'il a acquises dans les langues occidentales. On pourra discuter les choix proposés ici ou là, ce qui nous intéresse ici c'est l'ampleur de l'enjeu et l'impératif que représente la socialisation de ces techniques et donc des cohortes de termes qu'elles nécessitent. Si ces termes ne sont pas disponibles, ils doivent le devenir, que l'on procède par recension de créations spontanées, ou que l'on propose à l'usage des candidats termes. Après tout, les missionnaires ont bien dû, en leur temps, traduire dans les langues de leurs nouveaux fidèles les termes désignant la communion des saints, le Saint-Esprit, ou la transsubstantiation. Il n'est pas sûr que l'équipement du vocabulaire informatique représente une gageure plus difficile... (Gaudin 2007: 26)

$\mathrm{Au}$ «Forum international de Bamako sur le multilinguisme» de 2009, l'absence de littéracie dans les langues maternelles et les problèmes techniques non encore résolus pour créer du contenu localement ont été au centre des propos, mais avant d'aborder ces problèmes, nous allons évoquer la question de la nécessaire transposition culturelle impliquée par la localisation.

\subsubsection{Localisation: transposition culturelle}

Les particularités culturelles sont au cœur de la traduction, et la problématique de la localisation y est d'autant plus sensible que les écarts entre cultures sont importants. Pour traduire de et vers les langues africaines, il importe de prendre en compte les différences dans les codes d'interactions verbale et sociale des différentes cultures, 
qu'il s'agisse du passage entre LGC et langues africaines, ou bien du passage entre les différentes langues/cultures africaines elles-mêmes, dont les locuteurs font appel, dans la communication courante, à des ensembles spécifiques de stratégies où proverbes, maximes, paraboles, métaphores et images sont convoqués de manière originale. Les traducteurs ayant fréquemment à travailler sur des textes dont le fonds culturel n'est pas le leur, ils ne peuvent localiser de manière satisfaisante et accomplir leur tâche de transposition culturelle que si leurs compétences incluent la maîtrise des codes d'interaction des langues dans lesquelles ils travaillent.

Rabenero (2005) illustre bien l'ampleur de ces différences et les difficultés qu'elles présentent à propos de la traduction des manuels scolaires du français vers le malgache: les univers, les modes d'interaction et les logiques déployées dans les manuels français ne correspondent pas à ceux des destinataires malgaches. À ne pas suffisamment prendre en compte ces différences culturelles, le risque est, selon Rabenero, que leur langue devienne une langue étrangère à ses propres usagers.

Diki-Kidiri (2007a) présente une méthodologie détaillée et pragmatique de la terminologie culturelle dans le contexte du développement des langues africaines. Pour cet auteur, les questions de fidélité et d'équivalence fonctionnelle sont à reposer dans la perspective d'une approche culturelle de la terminologie. Une telle approche, dans chaque société concernée,

[p]ermet à cette société de trouver le mot juste pour exprimer chaque concept nouveau en puisant ses ressources linguistiques dans sa propre culture et selon sa propre perception du réel. En plaçant la culture au cœur de sa démarche, la terminologie culturelle se démarque nettement de la terminologie classique. (Diki-Kidiri 2007a: 14)

S'agissant de traduction dans le domaine des technologies de l'information, Ben Henda (2004) écrit, à propos des traductions vers les langues africaines:

On note également que les développeurs de logiciels et les fabricants de matériel informatique ne tiennent pas toujours bien compte, dans leurs produits, de certains aspects culturels, sociologiques ou même cognitifs importants pour les locuteurs du Sud. Par exemple, les logiciels d'origines latines, adaptés pour la langue arabe, présentent souvent un nombre assez important d'incohérences aux yeux de l'utilisateur arabophone type. Ces incohérences peuvent avoir de grandes répercussions sur l'acceptabilité, l'utilisabilité et l'efficacité même de l'interface. (Ben Henda 2004: 61)

S’il est réaliste de penser que la plupart des logiciels continueront de provenir des pays du Nord, ce même auteur espère cependant «que la localisation de ces outils sera faite, à l'avenir, par des personnes-ressources vivant dans le marché linguistique et culturel visé, plutôt que par des experts n'ayant qu'une connaissance théorique de la langue cible» (Ben Henda 2004: 62).

\subsection{Les aspects techniques de la traduction avec les technologies de l'information}

Comme commencent maintenant à le dire quelques-unes des personnes impliquées dans le développement de ressources numériques pour l'Afrique, il est beaucoup plus facile d'enseigner à un ordinateur à gérer une nouvelle langue que d'enseigner à des millions de personnes les langues que l'ordinateur connaît. 
Cependant le travail à réaliser est ici encore énorme et il concerne tous les niveaux de la traduction. Nous parlerons successivement ici de l'encodage des caractères, des outils du traducteur, et de la traduction/localisation de l'environnement logiciel.

Ben Henda (2004) parle de langues «technologiquement minoritaires», en ce sens que leur traitement informatique n'est pas très avancé par comparaison avec les langues latines. Il attribue une partie du problème au fait que pour un certain nombre de langues africaines, on est amené à gérer dans le même contexte logiciel ou applicatif des langues de familles différentes; on est en présence d'un multilinguisme «lourd», difficile à gérer par l'interface homme-machine et les systèmes internes de traitement des données codées à cause de la forme graphique des caractères (graphie latine, arabe ou autre graphie) et de la directionnalité de l'acte graphique (gauchedroite ou droite-gauche).

\subsubsection{L'encodage des caractères}

En 2004, Diki-Kidiri abordait déjà la question de l'encodage des caractères africains. Il rappelait que les logiciels capables de les prendre en considération étaient assez rares et insistait sur la nécessité de travailler à résoudre les nombreux problèmes techniques qui handicapent la normalisation, par le consortium Unicode, des caractères spéciaux nécessaires à l'écriture des langues africaines. Depuis, comme on le verra, du chemin a été fait sur la voie de l'encodage, mais les obstacles sont encore nombreux.

Pourtant, comme le soulignait Yang, de l'Office québécois de la langue française, lors de son intervention à la «Vitrine-forum sur l'informatisation des langues de Montréal» en 2003, c'est à la technique de s'adapter à l'être humain, pas le contraire:

La technique de la numérisation, les technologies informatiques ont été inventées par l'homme et peuvent évoluer selon les besoins de l'homme. Est-ce que nous devons nous borner à nous adapter à une technique datant d'une autre époque ou est-ce que nous devons chercher à adapter la technique à nos besoins? Les Coréens, entre autres, n'ont pas hésité à choisir la deuxième option.

L'écriture coréenne a été inventée au $\mathrm{Xv}^{\mathrm{e}}$ siècle et sa prise en compte en informatique était beaucoup plus compliquée au départ que celle du français. Pourtant les Coréens n'ont jamais songé à remplacer le Hangul par l'alphabet latin. En fait, ils se sont tellement battus pour une solution favorisant l'utilisation de leurs caractères et de leur graphie, que le consortium Unicode, que Microsoft, que l'ISO ont dû accepter de faire des changements dans les normes établies. Les Coréens ne voulaient pas renoncer à satisfaire leurs besoins culturels et linguistiques seulement parce que les techniques de codage du moment ne jouaient pas en leur faveur. Les pays du Sud n'ont pas à le faire davantage. (Yang 2004: 68)

Cependant, il est clair que les rapports de force ne sont pas les mêmes pour l'Afrique sub-saharienne, et comme le précise Lipou (2007), les ressources financières à mobiliser pour réaliser les projets d'extension de la norme Unicode à divers caractères de transcription sont incontestablement un frein et un filtre de sélection des systèmes graphiques et des documents africains à informatiser.

Dans une veine différente, Diki-Kidiri précise:

[I]l serait intéressant de tenir compte, lors des travaux d'informatisation des langues africaines, des écritures qui ont été utilisées pour rédiger certains des textes anciens 
de l'Afrique, comme le tifinar, le masaba, le nsibidi, et ainsi de suite. On ne le fait pas actuellement, avec pour résultat qu'un linguiste étudiant ces écritures ne peut pas les saisir dans ses documents de recherche. Il doit se contenter de faire des copier-coller d'images. (Diki-Kidiri 2004: 65)

\subsubsection{Les outils du traducteur}

Les traducteurs exercent aujourd'hui leurs activités dans de nouveaux contextes qui sont bien éloignés de ceux dans lesquels se réalisaient les traductions de la Bible des premiers missionnaires chrétiens en Afrique sub-saharienne. Les techniques et outils traditionnels de la traduction sont utilisés dans un espace mondialisé et s'appuient désormais sur les technologies de l'information. Les dictionnaires, lemmatiseurs et correcteurs pour les langues africaines sont en cours d'élaboration et d'informatisation, notamment dans certaines universités d'Afrique sub-saharienne (au Cameroun, au Kenya, au Nigeria et en République centrafricaine, notamment), mais ces travaux doivent encore avancer pour certaines des langues dans lesquelles standardisation et création terminologique sont récentes, et surtout être diffusés.

Par conséquent, il y a encore beaucoup à construire, notamment des bases de données terminologiques et des mémoires de traduction pour ces langues qui ont franchi les étapes de la description, de la standardisation et du développement terminologique, et plus encore pour les langues, encore nombreuses, qui ne les ont pas franchies.

Se pose aussi le problème de la ou des langues pivots. Par exemple, Ben Henda signalait en 2004 qu'il arrive souvent qu'il faille d'abord, pour traduire un texte depuis l'arabe, le traduire en anglais, parce qu'aucune passerelle directe n'existe entre l'arabe et le français. La situation a évolué depuis pour l'arabe, mais pour les langues d'Afrique sub-saharienne elle n'est pas meilleure, et les traducteurs des pays d'Afrique francophone, majoritaires dans la région, ne sont pas souvent formés pour fonctionner dans un environnement anglophone.

On trouve dans les «Actes de la vitrine-forum 2003» sur l'informatisation des langues de Montréal la présentation des produits récents et des prototypes existants à l'époque pour l'informatisation des données linguistiques et culturelles et la traduction. Notons que parmi les produits, les logiciels de gestion terminologique et de traduction ne prennent pas encore en charge les langues de l'Afrique sub-saharienne, mais que le plan d'action proposé inclut le développement et l'élargissement de l'Inventaire du traitement informatique des langues mis en place par l'Agence intergouvernementale de la Francophonie (AIF), aujourd'hui Organisation internationale de la Francophonie (OIF).

\subsubsection{La localisation de l'environnement logiciel}

Abordant la question de la localisation des logiciels les plus utilisés aujourd'hui (Microsoft Windows et Microsoft Office), Lipou souligne que les enjeux de rentabilité commerciale ne peuvent pas ne pas constituer un obstacle de taille pour les langues minoritaires: «[...] leur exclusion du cercle de celles dont les usagers jouissent de l'accès aux outils de la reconnaissance vocale ou de la traduction par informatique paraît inévitable» (Lipou 2007: 56). Les entreprises commerciales, il est vrai, font bien souvent l'impasse sur la localisation pour les «petites» langues, a fortiori si elles sont dotées de peu de prestige et de locuteurs peu nombreux. 
Pourtant, la production ou la localisation de logiciels de base en langues africaines est absolument nécessaire. Selon Diki-Kidiri:

[...] dans le Sud comme dans le Nord, on a aujourd'hui besoin de suites bureautiques pour être productifs. Cependant, ces outils indispensables ne sont pas, le plus souvent, disponibles dans la langue des personnes appelées à les utiliser pour produire de l'information. (Diki-Kidiri 2004: 65)

Même si des équipes sont prêtes à travailler à la localisation en langues africaines des logiciels libres utiles aux gens du Sud, il manque souvent les liens de collaboration nécessaires au succès de telles entreprises.

Comme le rappelle Osborn (2010), l'internationalisation et la localisation sont étroitement liées. Des organisations internationales comme l'Organisation internationale de normalisation (ISO), l'Internet Engineering Task Force (IETF) ou le World Wide Web Consortium (W3C) travaillent à l'élaboration des normes et des standards qui gouvernent le fonctionnement d'Internet et des ordinateurs qui permettent d'y accéder. L'IETF travaille à la prise en compte de langues et de scripts de plus en plus nombreux (par exemple, pour les noms de domaine, l'internationalisation des adresses de courrier électronique, etc.).

En outre, est encore à venir la mise en œuvre des paramètres régionaux, permettant aux systèmes informatiques d'identifier les données d'une langue et d'un pays, et préalable à toutes les activités de localisation pour une langue, de manière à ce que les documents puissent être identifiés par langue d'origine (facilitant des dispositifs tels que la recherche, la vérification d'orthographe, etc.).

Il faut aussi localiser les outils permettant aux internautes de faire de la navigation, de publier des documents sur la Toile, de correspondre par courrier électronique dans leurs langues, etc. Or, il reste encore à rendre les navigateurs capables de reconnaître les caractères spéciaux de certaines langues africaines (voir plus haut).

Diki-Kidiri (2007b) esquisse les stratégies susceptibles de préparer les voies d'accès des langues africaines au cyberespace en proposant des étapes de localisation et de traduction progressives:

Le site Internet est la meilleure façon d'affirmer la présence d'une langue dans le cyberespace, car on peut le décliner à l'infini, depuis la page personnelle jusqu'au portail multimédia offrant des services divers et variés. Avant de se lancer dans la création d'un site, il convient de prendre en compte les observations suivantes:

Un site écrit dans une langue peu dotée ne peut être lu que par les locuteurs de cette langue. Cela devient difficile si ces locuteurs ne savent pas lire et écrire dans leur langue. Le site peut alors servir à les y encourager. Quand on vient de mettre au point une orthographe pour la langue peu dotée, il est préférable d'envisager de créer un site bilingue utilisant simultanément la langue peu dotée et une langue de plus grande diffusion parlée dans la région. Dans ce cas, il faut absolument veiller à ce que la langue peu dotée soit présente dans les boutons et commandes de navigation ainsi que dans tous les messages destinés au visiteur du site afin d'éviter qu'elle ne se retrouve en position d'objet de curiosité et perde son statut de langue de travail sur la Toile. (DikiKidiri 2007b : 37)

À côté de la localisation sur la Toile, nous devons garder à l'esprit que le développement de la téléphonie mobile et les développements futurs de nouvelles applications apportent et apporteront des changements significatifs dans les usages, et donc exigent une certaine flexibilité dans la planification de la localisation. 
En réponse à la multiplication des besoins en traduction et localisation pour l'Afrique sub-saharienne dans un contexte de mondialisation et d'informatisation de la communication, des acteurs de types divers ont pris des initiatives d'envergures également diverses. Les actions s'intègrent donc dans un contexte où les niveaux d'intervention sont multiples: organisations internationales, gouvernements, entreprises privées, ONG, tissent un réseau complexe d'objectifs et de réponses aux problèmes à résoudre.

Pour faire état des travaux réalisés, de ceux qui sont en cours de réalisation et des problèmes qui se posent, nous allons présenter les principales prises de position et actions liées à la traduction et à la localisation en distinguant celles qui émanent d'organisations hors Afrique et celles qui émanent d'organisations en Afrique. Notons que les collaborations qui se tissent étant fréquentes, il devient parfois difficile de séparer nettement les unes des autres. Les travaux en cours dans le cadre du Réseau panafricain sur la localisation (ANLoc) méritent d'être présentés ensuite de manière plus détaillée.

\section{Les actions liées à la traduction et à la localisation}

Toutes les organisations œuvrant pour le développement du continent africain et travaillant dans le secteur de l'implantation des technologies numériques et de leurs applications ainsi que du développement de la connectivité sur le continent n'abordent pas la question des langues et de la nécessité de traduire et de localiser, loin s'en faut. L'impasse sur l'importance des langues maternelles et sur les besoins de traduction de et vers les langues africaines est plus souvent la règle que l'exception; comme on l'a vu à propos de eLearning Africa, cette impasse est souvent révélatrice de la prise de conscience de l'importance des obstacles à franchir. Nous mentionnons ici les actions officielles qui s'intéressent ouvertement au traitement des langues africaines.

\subsection{Les actions pilotées par des organisations hors de l'Afrique}

Les Nations Unies. Rappelons d'abord que l'Union internationale des télécommunications (UIT/ITU) ${ }^{9}$, institution spécialisée des Nations Unies pour les technologies de l'information et de la communication, pôle de convergence mondial où se retrouvent pouvoirs publics et secteur privé, a été le principal responsable de l'organisation du «Sommet mondial sur la société de l'information» (SMSI) de Tunis en 2005, dont les recommandations insistaient sur la nécessité

de faire progresser l'adoption du multilinguisme dans un certain nombre de secteurs: noms de domaine, adresses de courrier électronique, recherche par mot-clé;

de mettre en œuvre des programmes autorisant la présence de noms de domaine et de contenus multilingues sur l'Internet, et d'utiliser divers modèles logiciels pour faire face au problème de la fracture numérique linguistique et assurer la participation de tous dans la nouvelle société qui se fait jour. (Agenda de Tunis, Recommandations du SMSI 2005: 53; souligné par l'auteure)

L'UNESCO a mis en œuvre une politique linguistique mondiale fondée sur le plurilinguisme. Depuis la Recommandation sur la promotion et l'usage du multilinguisme et l'accès universel au cyberespace, adoptée en octobre 2003, l'UNESCO agit dans trois domaines: l'introduction de nouvelles langues sur Internet, la création et 
la diffusion de contenus en langues locales dans le cyberespace, l'accès multilingue aux ressources numériques. Le document «Comment assurer la présence d'une langue dans le cyberespace?» produit par l'UNESCO (Diki-Kidiri 2007b) appelle à la mobilisation pour soutenir l'introduction des langues "peu dotées» dans le cyberespace. Une nouvelle plateforme destinée à faciliter l'échange d'informations a été lancée (UNESCO [2008b] avec la ligne d'action C8 «Diversité Culturelle et Identité, Diversité Linguistique et Contenu Local»:

Le langage est le vecteur principal de transmission du savoir et des traditions. Ainsi, la possibilité d'utiliser son propre idiome sur les réseaux mondiaux d'information comme l'Internet déterminera le degré d'intégration d'un individu aux sociétés du savoir émergentes.

Actuellement, sur l'Internet, aucun contenu informatif n'est disponible dans les milliers des langues parlées dans le monde. Il n'existe pas non plus d'outil pour créer des contenus dans ces langues ou traduire des informations existantes dans ces idiomes. La manière d'accéder aux sites Internet par les noms de domaines est également limitée principalement à l'utilisation du script latin.

Des catégories entières de la population mondiale sont donc privées d'un accès équitable aux bénéfices produits par ces avancés technologiques et de l'obtention d'une information qui pourrait être essentielle à leur bien-être et leur développement. Si cette situation n'évolue pas, la diversité culturelle ne cessera de se réduire sur les réseaux d'information et les inégalités socio-économiques actuelles ne cesseront d'empirer.

En soutenant le développement d'un cyberespace multilingue, l'UNESCO promeut un accès plus large et plus équitable aux réseaux d'information et en même temps offre des possibilités pour la préservation des langues menacées de disparition à travers les TIC. (Sommet mondial sur la société de l'information 2003)

La Francophonie institutionnelle. Portée surtout par le Québec et la France, très concernés par la domination de l'anglais sur la Toile, la Francophonie institutionnelle joue un rôle important pour fournir à ses membres du Sud les ressources et la formation nécessaires à l'utilisation des technologies de l'information pour développer du contenu en français et - en principe - dans les langues partenaires.

La Francophonie institutionnelle a de plus en plus recours à Internet, d'où la nécessité renouvelée de passerelles entre le français et les langues partenaires, et entre toutes les langues dans une société globalisée. Ses structures ${ }^{10}$ ont été historiquement dédiées exclusivement à des contenus francophones. Ce n'est que très récemment que de nouvelles déclarations concernant la nécessaire prise en compte des langues nationales ont été formulées pour le développement des applications contribuant à renforcer la présence du français et des langues partenaires sur Internet.

Notons par exemple, qu'au sein du RIFAL, l'Office québécois de la langue française a développé une base de données terminologiques et textuelles multilingue et multi-plateforme, qui tiendra compte des spécificités du tri alphabétique, tonal et structurel du lingala et du hausa, langues transfrontalières d'Afrique de l'Ouest et d'Afrique centrale.

Le Centre de recherches pour le développement international (CRDI), un organisme canadien déjà mentionné, a instauré en 1997 le programme Acacia, une initiative d'appui à l'Afrique sub-saharienne pour mettre les TIC au service de leur développement social et économique. L'année 2007 a vu la création d'un réseau africain consacré à la localisation des TIC en langues africaines ${ }^{11}$. Les objectifs en matière de développement mettent l'accent sur le caractère crucial, pour l'adoption 
des TIC et l'utilisation optimale des ordinateurs et d'Internet, de la disponibilité de logiciels et de contenus dans la ou les langues connues des utilisateurs.

Le groupe de recherche Research ICT Africa (RIA) a été financé également par le CRDI pour combler un vide stratégique dans le développement d'une société de l'information durable: cette structure s'intéresse en particulier à l'étude des compétences requises pour une utilisation efficace d'Internet - ce qui touche à la compétence langagière, et à la définition du terme contenu local.

La Société internationale de linguistique (SIL) est une organisation qui travaille depuis plusieurs décennies dans le domaine des langues les moins diffusées des pays du Sud. Elle a réalisé récemment le site Ethnologue.com, qui reflète les résultats de ses travaux et fait aujourd'hui autorité. On peut y trouver des ressources très fournies sur les langues du monde les moins diffusées, et en particulier sur les langues d'Afrique sub-saharienne: bases de données, cartes linguistiques, bibliographies, logiciels, polices de caractères...

FUNREDES, Linguamón. Bien que leur activité ne soit pas exclusivement centrée sur l'Afrique, ces deux organisations poursuivent les mêmes objectifs de sauvegarde de la diversité linguistique. FUNREDES est une organisation non gouvernementale (ONG) internationale qui se consacre à la diffusion des TIC dans les pays en développement, notamment en Amérique latine et dans les Caraïbes. Sa réalisation principale a été de mesurer la répartition des ressources Internet en termes de diversité linguistique: internautes, pages web, courriels, etc. Linguamón Casa de les Llengües («Maison des langues») ${ }^{12}$ est un consortium formé à l'origine par le gouvernement de la Catalogne, le Centre UNESCO de la Catalogne et la Fondation Forum universel des cultures. L'objectif de ses membres est la promotion de la diversité linguistique. Avec le Réseau Maaya (voir sous-section suivante), Linguamón a organisé fin septembre 2009 à Barcelone le «Symposium international sur le multilinguisme dans le cyberespace: les sociétés de la connaissance peuvent-elles se permettre de ne pas être multilingues?» Il s'agissait de mener une réflexion sur les enjeux de la diversité linguistique dans Internet afin de définir les priorités d'actions et de politiques en faveur d'une présence accrue des langues dans le cyberespace. Ces deux organisations agissent comme facilitateurs et fédérateurs d'initiatives en matière de traduction et de localisation.

\subsection{Les actions africaines}

Le Centre sur les politiques internationales des TIC Afrique du Centre et de l'Ouest (CIPACO), mis en place par l'Institut Panos Afrique de l'Ouest (IPAO) ${ }^{13}$, est une ONG africaine dont l'objectif est de promouvoir une culture de la démocratie, de la citoyenneté et de la paix, à travers l'information et la communication. Il a pour but de renforcer les capacités des acteurs en Afrique de l'Ouest et du Centre pour une meilleure participation à la prise de décision internationale en matière de TIC.

Le site du CIPACO, en anglais, français et portugais, fait état de l'enjeu de la préservation et de la promotion des langues nationales face aux langues officielles d'emprunt ou des langues minoritaires face aux ensembles ethnolinguistiques dominants ${ }^{14}$. On peut y lire aussi que les décisions internationales qui sont prises en ce domaine ne reflètent pas souvent la diversité des situations et des points de vue et 
qu'il faut développer les identités culturelles, et donc localiser, traduire, créer des contenus en langues africaines.

Cette prise de position contraste avec celle de la politique linguistique de la Francophonie, qui, tout en encourageant officiellement la production de contenus multilingues, met l'accent sur la valorisation et le partage des contenus numériques, documentaires et archivistiques francophones - politique linguistique qui subit ellemême cette tension dont nous parlions plus tôt: assurer la visibilité du français face à l'anglais (ce qui est une certaine déclinaison de la diversité sur la Toile), donc développer l'accès et des contenus francophones, d'une part; et, d'autre part, respecter la diversité culturelle, et donc traduire et localiser.

L'Académie Africaine des Langues (ACALAN), créée en 2000 et implantée à Bamako (Mali), met en œuvre une coopération scientifique et technique interafricaine. Elle a lancé en 2006 le projet Langues africaines et Cyberespace piloté par un consortium qui comprend deux acteurs majeurs africains spécialisés dans le domaine des TIC, l'Institut Meraka (Afrique du Sud) et l'Institut Panos Afrique de l'Ouest (Sénégal). Il doit permettre aux langues africaines d'être «capacitées» (formulation utilisée par l'ACALAN), puis de pouvoir être prises en charge dans le système éducatif.

Le site Internet de l'ACALAN, aujourd'hui très riche et structuré, est de création récente. Il propose des liens vers un grand nombre d'autres sites sur les langues africaines, leurs systèmes d'écriture, les travaux terminologiques et d'encodage. La page Le Web des linguistes fournit une liste des sites qui parlent des langues africaines et redirige vers des sites en langues africaines (mais on trouve parmi ceux-ci peu de langues sub-sahariennes).

L'African Languages Technology Initiative (Alt-i), initiative nigériane, a été mise en place pour faciliter le développement des ressources qui permettront aux langues africaines de s'engager dans les technologies de l'information. Cette organisation met l'accent sur la nécessité de permettre aux Africains de communiquer au moyen des machines et avec les machines dans leurs langues: claviers, logiciels, ressources permettant de remettre l'oralité à l'honneur (reconnaissance de la parole, synthèse vocale). Les travaux sont en cours pour le yoruba (langue nigériane et transfrontalière); notamment, deux propositions de claviers localisés pour le yoruba ont été récemment soumises aux utilisateurs. Cette organisation insiste aussi sur l'importance des travaux à réaliser dans le domaine de la traduction automatique.

Le Réseau Maaya a été créé dans le cadre du SMSI. Il a été mis sur pied par l'ACALAN, sous les auspices de l'Union africaine. En 2008, dans le cadre de la promotion du multilinguisme et de la diversité linguistique dans le cyberespace, le Réseau Maaya a réalisé le projet Voix et textes, qui a pour ambition de faire en sorte que l'oralité soit revalorisée à côté de l'écrit. L'objectif final du projet est de développer un système de traitement de données multilingues orales et textuelles pour les langues africaines. Ce système doit pouvoir servir de base technologique à de nombreuses applications en reconnaissance automatique de la parole, en traduction automatique ou assistée par ordinateur et en synthèse vocale et tonale, pour les langues africaines qui sont peu dotées en ressources numériques, alors qu'elles disposent d'importants patrimoines immatériels qu'il est urgent de sauvegarder. Parmi ses objectifs figurent l'égal accès de toutes les langues au cyberespace et la promotion de la localisation des logiciels. 
Translate.org est une ONG sud-africaine travaillant sur la localisation de logiciels libres et en code source libre (Free and Open Source Software, FOSS) en langues africaines. Son travail a conduit au développement de la première version du logiciel OpenOffice en langue africaine. Celui-ci est maintenant disponible dans les 11 langues officielles d'Afrique du Sud, et y ont été ajoutés aujourd'hui Mozilla Firefox, Mozilla Thunderbird, et des portions de GNOME et KDE. Dans les activités de cette organisation, l'effort porte sur l'élimination des barrières techniques qui ont un impact négatif sur la langue. Ainsi, il y a eu création d'un clavier sud-africain qui inclut tous les caractères nécessaires pour écrire dans les 11 langues officielles, y compris des polices adaptées pour écrire en venda.

Des localiseurs compétents issus de cette organisation se sont implantés ailleurs en Afrique et dans le monde. Des offres de collaboration avec les pays d'Afrique subsaharienne ont été lancées. En effet, tout en étant focalisés sur les langues d'Afrique du Sud, les travaux de Translate.org contribuent à faire avancer ceux pour les autres langues africaines (stratégies, caractères développés, etc.)

Le réseau éthiopien Free and Open Source Software (EFOSSNet) est une entreprise non gouvernementale établie en 2005 dont l'objectif est de créer un environnement d'utilisation des logiciels en code source libre en langues éthiopiennes. Encore une fois, les initiatives et créations de ressources endogènes en un lieu ou un autre de l'Afrique sub-saharienne contribuent à faire progresser la localisation pour l'ensemble des langues africaines.

Des organisations ou des entreprises comme IT+46, Bisharat, Kamusi, Kasahorow, OpenCraft, se sont mobilisées pour assurer le développement durable par les technologies de l'information. La plupart de nature collaborative, elles travaillent à l'élaboration de ressources de base pour la traduction ou la localisation afin de permettre l'utilisation des langues africaines dans les logiciels et les contenus sur la Toile: par exemple, création de dictionnaires unilingues et bilingues et de ressources d'apprentissage, standards techniques pour les langues africaines, architecture des systèmes pour le Web 2.0 en code source libre, notamment pour la préservation et la modernisation de l'héritage culturel local. Bisharat met en avant l'incongruité de la réalité courante en Afrique en ce qui concerne la production des connaissances, jusqu'ici organisée à partir de langues que peu de personnes parlent, et encore moins lisent ou écrivent. On trouve sur son site des pages de démonstration sur des sujets divers employant des langues africaines, des explications sur la nature et les problèmes de la traduction automatique, et sur ce qui serait possible s'il y avait des traducteurs pour les langues de l'Afrique. En 2007, le site était seulement en anglais et en français, il est maintenant en anglais, français, portugais et hausa. On trouve sur la page A12n Gateway / Portail A12N ${ }^{15}$ des groupes de discussion et pages de référence pour l'encodage, polices et claviers pour langues africaines. Quant aux forums de Bisharat sur les langues africaines, ils proposent des sujets de discussion sur les questions techniques d'orthographe, de standardisation, par exemple pour les langues du Togo, du Burkina Faso, du Ghana, du Bénin, ou du Sénégal. On y discute de claviers et de polices de caractères.

La Faculté d'informatique de l'Université de Nairobi, Kenya, est engagée dans la recherche en ingénierie linguistique: plusieurs langues locales ont été ciblées: swahili, kikuyu, dholuo, kikamba et ekegussi. Des données ont été recueillies pour chacune d'elles; des traitements de texte et des traducteurs automatiques ont été développés. 
Ce centre de recherche travaille aussi sur des applications localisées pour les téléphones mobiles.

Le rapport général produit à l'issue de la conférence régionale de 2007 à Yaoundé recommandait la mise en application de toutes les décisions prises en faveur de la promotion des langues africaines par les grandes organisations internationales. Ce rapport réaffirmait notamment la nécessité de la codification de toutes les langues sur le plan national (tâches des structures nationales chargées de la promotion des langues nationales), la localisation des logiciels libres ou non dans les langues nationales, l'organisation de l'édition en langues africaines par la coédition et l'édition multilingue d'ouvrages traduits, la création terminologique pour tous les secteurs d'activité. Quatre grands projets à soutenir ont émergé de cette conférence:

- Le Centre panafricain d'interprétation et de traduction, basé au siège de l'ACALAN à Bamako, doit permettre de créer les conditions idoines de formation, d'interprétation et de traduction dans les langues de travail de l'Union africaine en général et dans les langues africaines transfrontalières en particulier;

- Le Projet de terminologie basé à Dar-Es-Salam, Tanzanie, a pour but de mieux assurer la capacitation [sic] des langues africaines et renforcer le processus d'harmonisation des concepts;

- Le Projet "Langues africaines et Cyberespace», basé à Addis-Abeba, Éthiopie, a la charge de garantir l'utilisation des langues africaines dans le cyberespace;

- Le Projet de lexicographie, basé à Gaborone, Botswana, permettra de doter les langues africaines des instruments lexicographiques indispensables à leur valorisation.

En outre, à partir du plan d'action du SMSI, la Commission économique pour l'Afrique (CEA) a envoyé un questionnaire à toutes les infrastructures nationales de l'information et de la communication et à tous les points focaux nationaux du SMSI dans les États membres et les communautés économiques régionales, pour évaluer leur engagement dans la mise en œuvre du plan d'action. L'analyse des résultats de ce questionnaire a été publiée dans un rapport de 2009. Pour ce qui concerne le développement de contenus locaux, les résultats pays par pays font état d'avancées non négligeables, qui sont présentées comme suit:

- Éthiopie

Un projet d'adaptation aux besoins locaux concernant l'établissement de glossaires de terminologie des TIC pour trois langues locales a été mené à bien et un autre est en cours d'exécution pour deux autres langues locales. Un clavier standard a été mis au point pour l'alphabet éthiopien. [...] [u]n logiciel et des normes ont été développés pour un ensemble de caractères éthiopiens.

- Madagascar

Il y a un vaste projet visant à adapter les TIC au contexte de Madagascar.

Le logiciel qui est utilisé pour le développement communautaire a été adapté aux besoins locaux.

- Mali

Un projet sur la numérisation des anciens manuscrits de Tombouctou est en cours d'exécution.

Soutien à la création de logiciels dans les langues locales.

Un programme de logiciels en langue bambara a été développé dans le cadre du dictionnaire multilingue (DIDADI) dont un assistant d'université est en train de s'occuper. 
- Sénégal

Microsoft a commencé la traduction de Windows Vista et de Microsoft Office 2007 en wolof. Un dictionnaire informatique français-wolof est en cours d'élaboration dans le cadre d'un projet appelé ANAFA.

- Nigeria

Il y a des initiatives pour créer des logiciels dans trois langues principales locales (Hausa, Yoruba et Ibo). Il y a également des partenariats public-privé avec Microsoft, la société civile, les organisations privées et établissements de recherche locaux pour appuyer des programmes de recherche-développement concernant le développement de matériel et de logiciels.

Ce rapport montre certes que les travaux sont en cours, mais aussi que les tâches restant à réaliser nécessitent encore un investissement considérable pour répondre aux besoins en traduction et localisation et pour couvrir un plus grand nombre de langues.

\subsection{ANLoc}

Le Réseau panafricain pour la localisation a pris le relais de PanafrL10N, dont l'objectif, selon les termes idéalistes de Yacob (2004), était de «faire de l'ordinateur une extension naturelle de la société qu'il sert». Cette organisation a pour objectif de produire des dictionnaires, des terminologies et des paramètres régionaux pour les logiciels. Elle soutient aussi la formation de traducteurs de logiciels en langues africaines (en collaboration avec le Centre de recherche sur la localisation de Limerick, Irlande).

ANLoc se décline aujourd'hui en plusieurs projets. Son site Internet a récemment évolué: tout en restant un lieu de ressources pour les localiseurs, il fait maintenant usage de la technologie wiki pour continuer à rassembler des informations dans un format ouvert et flexible et pour faciliter l'interaction entre les communautés de localisation.

Parmi les projets du Réseau panafricain pour la localisation, Afrigen ${ }^{16}$, lancé en septembre 2009, a pour objectif de créer des paramètres régionaux (anglais: locales) pour 100 langues africaines dans un délai de 12 mois (à sa création, seulement 36 des 2000 langues africaines avaient leurs propres paramètres régionaux). Après avoir identifié environ 170 langues parlées par plus de 500000 locuteurs - à partir des estimations d'Ethnologue.com -, ANLoc les a groupées en huit zones sur le continent africain. Ensuite, dans un premier temps, les porteurs du projet ont demandé à des locuteurs natifs des langues cibles de travailler bénévolement pour créer des paramètres régionaux pour 100 de ces langues. On peut suivre sur le sitel'état d'avancement de la création des paramètres régionaux pour chacune des langues concernées. L'interface pour construire les paramètres régionaux dans une langue africaine donnée est disponible en anglais, français et swahili.

Un autre des projets actuels d'ANLoc s'intitule Outils de localisation, et vise à permettre aux traducteurs de faire leur travail, ainsi que de choisir la terminologie correcte. À l'intérieur de ce projet, le sous-projet Clavier a pour objectif de créer des claviers pour des groupes de langues africaines, de façon à ce que toutes puissent être écrites avec un ordinateur. Il doit aussi faire en sorte que ces claviers soient intégrés dans les logiciels ou bien facilement téléchargeables. Le sous-projet Polices a pour objectif de créer des outils pour mesurer la couverture africaine d'une police, de 
manière à pouvoir rechercher des polices apportant une couverture complète des scripts africains. Le sous-projet Localiser les logiciels s'intéresse à l'existant en open source en langues africaines, de façon à tout mettre en œuvre pour étendre la couverture des langues africaines. Un wiki est disponible pour permettre le partage d'information. (L'organisation non gouvernementale sud-africaine Translate.org participe à ce sous-projet.)

Le sous-projet Correcteurs d'orthographe s'intéresse à une question qui touche à la qualité de la localisation et qui est aussi une affaire de vitalité pour les langues. Il s'agit de construire des correcteurs d'orthographe et de diacritiques en prenant en compte les interrelations entre les langues africaines.

Le sous-projet Terminologie a pour objectif de créer une liste de 2500 mots pour utilisation par les localiseurs. Il doit aussi gérer les logiciels de terminologie pour faciliter la collaboration dans la production de terminologies dans tous les secteurs.

Enfin, les sous-projets Activités en réseau et Formation ont pour objectif de rassembler et de former les personnes travaillant à la localisation en langues africaines, avec des ateliers et des modules couvrant les besoins de localisation locaux.

On constate donc actuellement une convergence dans les objectifs de ces associations et organisations et une certaine synergie entre elles par le réseautage, pour que la technologie numérique fournisse un environnement favorable pour le plus grand nombre de langues.

\section{4. État des lieux et dynamique}

\subsection{La présence des langues sur la Toile et dans l'environnement logiciel}

On trouve sur la Toile de nombreux sites spécialisés dans la discussion et l'information sur des sujets africains, on y parle de l'Afrique et parfois des langues africaines, beaucoup moins dans ces langues. Si les contenus visent les Africains, en Afrique sub-saharienne ou en diasporas, ils fonctionnent en anglais ou français en grande majorité. Pour ne citer que quelques exemples, AllAfrica est un site en anglais et en français alimenté quotidiennement qui collecte et indexe du contenu provenant de plus de 125 organes de presse d'Afrique ainsi que de plus de 200 autres sources d'informations. Il donne des informations et propose des liens vers les principaux organes de presse dans chaque pays africain, en particulier à propos de la progression de la connectivité et des usages des TIC en Afrique. On y trouve les informations les plus récentes sur la connectivité, ses problèmes, les initiatives; très peu de choses sur les questions de langues.

Si l'on regarde du côté des initiatives assorties de dispositifs de communication sur la Toile (wikis, blogues ou forums), caractéristiques des pratiques avec la Toile au $\mathrm{XxI}^{\mathrm{e}}$ siècle, on trouve aussi surtout des ressources et des échanges ayant recours à l'anglais ou au français, qu'elles soient financées localement, régionalement, ou par la communauté internationale. Ainsi, Grioo.com, en français, est aujourd'hui le principal portail Internet dédié à la communauté noire francophone, avec une présence internationale des internautes; c'est aussi 60000 inscrits à la lettre d'information hebdomadaire, 3090 membres dans le forum. Il n'est pas question de langues africaines sur ce site. Afrik.com est un journal en ligne en français créé en 2000 par des journalistes issus de la presse écrite, de la radio et de la télévision; une édition quo- 
tidienne a été développée et déclinée sur 52 portails pays, ainsi que des rubriques thématiques telles que l'économie, la culture, la musique, les voyages, la beauté, l'art. Aujourd'hui, le site a une audience mensuelle d'un demi-million d'internautes et quatre millions de pages vues. Son forum, relativement actif (25 sujets, plus de 2100 membres inscrits et plus de 20000 messages), voit une moyenne d'une vingtaine d'utilisateurs connectés par jour; pendant l'une de nos connexions, nous avons observé 371 connexions simultanées. Sur le forum Speakafrica, plateforme d'échanges pour la jeunesse africaine, les pages sont en anglais et en français exclusivement.

Nous voyons donc que la communication en ligne s'est développée, et que le potentiel d'utilisateurs est bien là. Cependant, les forums de discussion utilisant exclusivement une langue d'Afrique sub-saharienne sont très peu nombreux. Les blogues africains, qui témoignent de l'appropriation de la Toile par les usagers et de la présence de cette région du monde dans le cyberespace, sont très nombreux; la plupart sont en anglais, et quelques-uns proposent un choix de langues pour lire et communiquer - ainsi que des outils de traduction, comme sur un site en swahili, qui propose un choix de dix-huit langues (mais pas d'autre langue d'Afrique sub-saharienne). Très majoritairement, les blogs sont produits par la diaspora africaine, qui manifeste aussi sa présence dans les autres outils de communication sur la Toile. La question du rôle de la diaspora dans le développement (développement économique et développement de l'information et de la communication) et la question du lien entre la diaspora et le pays d'origine sont ici d'une grande pertinence pour la réflexion sur la traduction et la localisation dans tous ces types de communication interactive sur la Toile.

BlogAfrica est justement un lieu où est abordée cette question. Il s'agit d'un portail de blogues africains, principalement en anglais. Quant aux sites francophones africains, les forums de discussion en particulier, on peut y remarquer un brassage des langues africaines et du français, reflet du va-et-vient entre l'attachement à la culture africaine et à l'Afrique, et à l'espace francophone; la cohabitation culturelle y est manifeste: on rencontre de plus en plus de sites sur les proverbes ou les contes africains, ou encore sur les prénoms africains; la langue utilisée y recourt abondamment aux emprunts aux langues africaines (daba, moutête, malafoutier...) et aux transferts. Certains sites associent des éléments des langues locales à la présentation d'informations touristiques ou culturelles. On y voit aussi un usage particulier d'Internet, qui consiste à mettre cet outil au service de la mémoire, comme cela a été le cas pour le site Afrocom qui hébergeait les littératures orales africaines. (Ce site ne fonctionne pas actuellement.) Mais il n'y a pas, c'est la règle, de localisation de l'environnement logiciel.

Cependant, un certain nombre de réalisations et de sites ont vu le jour au cours des dernières années pour donner vie aux langues africaines sur la Toile. La durée de vie de la plupart d'entre eux a été courte, mais leur simple existence, même éphémère, témoigne du potentiel et de la volonté de localiser et de traduire dans toutes les langues parlées en Afrique sub-saharienne. Par exemple, MailAfrica, créé en 2003, avait pour objectif de développer des interfaces de courriel dans 257 langues africaines estimées "principales» au cours des cinq années suivantes.

Online Formapro, en 2007, en collaboration avec le gouvernement du Sénégal et son initiative de solidarité numérique (action aujourd'hui terminée), a produit un 
tutoriel interactif utilisable en français, arabe, ou wolof sur le maniement de la souris: avec la Caravane solidarité numérique, un autobus équipé avec des ordinateurs (cyberbus) allait de village en village pour donner une première expérience de l'informatique aux habitants; par la suite, chaque village devait être doté d'un cyber-centre pour l'informatique, les communications, l'accès à Internet et enfin, l'alphabétisation de la population.

Le récent site Sango ti Be Africa (Sango de Centrafrique) est un exemple de ce qui peut être réalisé pour des langues comme le sango, qui a été instrumentalisé pour les environnements informatisés. Ce site est bilingue, et la localisation a été réalisée de manière à permettre la lecture dans les deux langues, français et sango.

Par ailleurs, le site Fulfulde.com, mis en ligne en 2002 pour faire connaître le fulfulde, langue transfrontalière utilisée du Sénégal jusqu'au Nord Cameroun, a été abandonné, et la page de Wikipédia traitant de cette langue est aujourd'hui au point mort, et ce, depuis plusieurs mois. Parmi la trentaine de langues africaines ayant des contenus sur Wikipédia, seulement sept sont des langues d'Afrique sub-saharienne.

Dans cette même veine, le projet WikiAfrica, lancé en 2006 et parti d'Italie, a effectivement pris l'option de publier ses contenus sur l'Afrique en langue italienne, en raison de la faiblesse et du caractère lacunaire des contenus traduits dans des langues africaines; ce choix se discute, mais l'intention était de produire des articles clés capables de susciter la curiosité et de fournir un matériau intéressant ${ }^{17}$.

Ouvert depuis fin 2002, le site Afrilivres ${ }^{18}$ illustre la co-existence et le renforcement mutuel des initiatives de traduction traditionnelle et de l'utilisation des technologies de l'information. Il présente un cyber-catalogue des titres non scolaires publiés par les éditeurs d'Afrique francophone regroupés en une association du même nom basée à Cotonou. On y trouve des titres d'ouvrages en français et bilingues en langues africaines et français (par exemple: Proverbes Murum du Tchad, Contes Dioula du Cameroun).

Du côté des logiciels, on trouve sur le site de Microsoft la liste des langues africaines - peu nombreuses - dans lesquelles Windows et ses logiciels ont été traduits; les langues d'Afrique sub-saharienne se comptent sur les doigts d'une main, celles d'Afrique du Sud sont au nombre de cinq également. En ce qui concerne les logiciels libres localisés, leur présence reste largement confidentielle dans l'attente de la diffusion et de la mise en commun des travaux menés en plusieurs lieux dont il a été question plus haut.

Comme nous l'avons dit, les acteurs sociaux qui influent sur les pratiques langagières en matière de traduction et de localisation sont positionnés à différents niveaux d'intervention. Et comme l'écrit Rousseau (2007), les organismes à vocation linguistique n'ont pas toujours le rôle ni le pouvoir d'influence que l'on croit; en face d'eux, l'ensemble des catégories d'acteurs, officiels ou non, dans la société - gouvernements, administration et services publics, clients et fournisseurs, publicitaires médias... -, contribuent par leurs attitudes et comportements langagiers (recours aux traductions et aux traducteurs, localisation ou non) à façonner l'usage des langues et influencent le marché. 


\subsection{Ressources en traduction}

On trouve sur la Toile de nombreux sites dédiés à la traduction ou comportant un espace d'aide à la traduction (dictionnaires en ligne, mémoires de traduction, traducteurs semi-automatiques, constitution automatique de lexiques et bases terminologiques bilingues, maintenance de corpus bilingues alignés et annotés, etc.).

Cependant, chez Translated, qui se déclare le seul prestataire de service au monde disposant de traducteurs natifs dans 107 pays, les seules langues représentées pour l'Afrique sub-saharienne sont le swahili, le kirundi, le tigrinya. Sur des sites proposant dictionnaires et/ou traductions, comme Lexicool, on ne trouve pour cette région du monde que le somali, le swahili et le wolof; sur les sites uniquement de traduction, très peu de langues d'Afrique subsaharienne. Plusieurs sites comportent un espace traduction, par exemple Sorosoro, mais les plateformes de traduction et de localisation de logiciels libres ou de gestion de ressources de traduction comme Launchpad ou Wordbee ne mentionnent pas les langues d'Afrique sub-saharienne.

Freelang est un projet à but non lucratif dont la création remonte à 1997. Les services proposés sont gratuits: les dictionnaires sont présentés par ordre alphabétique, ou par familles de langues, et peuvent être téléchargés gratuitement; 181 dictionnaires sont actuellement proposés, dont 7 pour des langues d'Afrique sub-saharienne. On y trouve aussi des logiciels de traduction, par système et par langue. Sur Freelang, on peut ajouter des mots dans les dictionnaires, on peut demander de l'aide par courriel pour des traductions courtes à but non commercial, et le service d'aide à la traduction permet de contacter un traducteur bénévole et d'obtenir une réponse par courriel, gratuitement. On peut aussi s'inscrire en tant que traducteur. Un forum est disponible pour des traductions ou pour des questions d'ordre général, sur les langues, sur le site ou sur tout autre sujet.

\subsection{La dynamique: facilitation et complexification}

La dynamique de la traduction et de la localisation se déploie dans des contextes de communication de plus en plus médiatisée par les technologies de l'information où la place des nombreuses langues d'Afrique sub-saharienne est fragile, et l'observation des actions et des réalisations pour cette région du monde révèle des processus et des pratiques qui agissent selon deux axes, l'un de facilitation et l'autre de complexification.

Du côté de la facilitation, il est permis de dire que toutes les avancées dans le domaine de la traduction et de la localisation - qu'il s'agisse de réalisations pour la construction d'outils de base ou bien de production de contenus localisés - facilitent les avancées futures pour la langue concernée et pour les autres langues de la région. En effet, toute visibilité ajoutée aux langues de moindre diffusion et prestige est susceptible de jouer un rôle de moteur pour la poursuite des travaux et développe le potentiel de création des traducteurs et localiseurs actuels et futurs; et il est clair que tout acquis pour une ou plusieurs langues sur le plan des outils de traduction et des outils de localisation contribue à faciliter le travail pour les autres langues en constituant un fonds de données et un ensemble de schémas conducteurs.

Cette visibilité émergente donne donc une nouvelle légitimité aux langues, et peut entraîner de la part de divers acteurs sociaux la mise en place de décisions 
construisant une capacité de développer des contenus aux niveaux locaux pour des langues ayant peu de locuteurs et/ou une diffusion très limitée: de plus, si outils et contenus sont créés, se créent aussi progressivement motivation et adoption de leur utilisation, comme en témoigne l'exemple des langues d'Afrique du Sud.

Et puisque les technologies de l'information permettent maintenant l'introduction, à côté des sites Web "classiques», de nouveaux types de sites collaboratifs et dialogiques (le Web 2.0) comme les forums, blogues et wikis, elles facilitent le fonctionnement de réseaux informels ou formels : la communication horizontale et bidirectionnelle et les initiatives et la créativité locales peuvent être exploitées pour développer la localisation; et les différents acteurs sociaux peuvent aussi plus aisément communiquer et collaborer. Cela permet, par exemple, de solliciter la traduction d'éléments de contenus spécifiques, la collaboration entre localisateurs, entre producteurs de contenus (diasporas, ou d'un pays à l'autre), le fonctionnement de réseaux de services pour la localisation, l'accès à des ressources de base ou des ressources pour la traduction ou la localisation de logiciels ou de contenus situées en des lieux éloignées géographiquement. Ajoutons que l'appropriation de ces environnements interactifs par un nombre croissant de personnes donne à celles-ci capacité de principe et légitimité pour communiquer - et par conséquent facilite le pas vers davantage de localisation, tant sur les sites institutionnels que sur les sites privés.

Ensuite, le rôle de la multimodalité dans la production de contenus localisés ne doit pas être sous-estimé: la localisation pour les langues/cultures à tradition orale peut s'appuyer sur des contenus multimodaux; et le transfert des matériaux culturels vers des supports numérisés, notamment en vue de construire la mémoire collective et d'en permettre la diffusion, peut donc se faire aujourd'hui de manière relativement satisfaisante pour les langues à corpus oral: par exemple, YouTube présente d'ores et déjà des contenus en des langues peu diffusées avec des traductions disponibles; on peut enregistrer de l'oral (récits, contes, proverbes, chansons, poèmes, etc.) et en solliciter des traductions orales ou écrites ${ }^{19}$.

Enfin, la démocratisation de la téléphonie mobile a contribué à modifier les comportements sociaux et à élargir les espaces dans lesquels se déroulent les pratiques langagières pour les populations de tous les âges et couches sociales.

Du côté de la complexification, plusieurs dimensions sont à considérer. Tout d'abord, les moyens de facilitation de la localisation et de la traduction, et plus généralement de la communication multilingue, se situent dans une configuration multipolaire. D'une part, avec les environnements informatisés, la traduction est faite aujourd'hui par des professionnels, certes, mais aussi avec la collaboration de réseaux informels de non-professionnels; elle peut être partielle, spécialisée, sur demande... D’autre part, on voit aussi que la multipolarité prévaut également du côté de la production des ressources, des informations, et même des formations; dans la gestion de la localisation à l'échelle africaine, leur mise à la disposition s'effectue à partir de multiples sources de nature et d'importance diverses, situées aussi en de multiples lieux matériels.

L'alternance codique (code switching) rendue possible aujourd'hui est une autre dimension de la complexification. On peut gérer sur Internet cette pratique courante des bilingues. Les environnements informatisés peuvent en effet proposer de nouveaux types d'aide multilingue, mais la structuration de l'alternance entre les langues sur un même site est à construire, et la gestion du passage d'une langue à l'autre est 
techniquement plus lourde que la simple redirection de l'utilisateur vers des pages dans une autre langue.

Les services pour la localisation sont aujourd'hui le résultat d'initiatives personnelles et institutionnelles; pour éviter la dispersion de ces efforts, le réseautage est une entreprise d'autant plus complexe que les moyens des unes et des autres sont souvent sans commune mesure. Pourtant, dans les institutions, réseaux régionaux, nationaux, internationaux, capables de produire des contenus localisés ou d'orienter les demandeurs vers des dispositifs appropriés, il peut sembler réaliste de concevoir l'existence de webmestres internationaux ayant des compétences dans des bouquets de langues, qui seraient capables de diriger les utilisateurs vers les ressources localisées ou permettant la localisation.

La recherche de solutions aux problèmes de localisation sur la Toile fait intervenir un ensemble complexe d'acteurs spécialisés, d'autres acteurs sociaux non spécialisés, de décisions politiques et de choix de mise en œuvre techniques. La combinaison de différents types de ressources constitue elle-même une opération complexe d'assemblage.

\section{Résumé et perspectives}

Les activités de traduction et de localisation s'organisent pour les langues de l'Afrique sub-saharienne dans des contextes de contacts linguistiques et culturels inégalitaires qui ont été un frein à leur déploiement. Le développement des technologies de l'information a contribué à refaçonner les rapports de force, et leur potentiel se conjugue aujourd'hui aux apports du Web 2.0, avec son éventail de dispositifs de communication et de collaboration multimodaux, et à ceux de nouvelles applications, pour apporter des éléments de solution et des ébauches de réponse dans des paysages très multilingues et techniquement sous-équipés.

Pour qui adopte une posture pessimiste, lourdeur et lenteur, motivations mitigées ou contrariées pourraient caractériser la problématique de la localisation dans cette région du monde. Si l'on opte pour l'optimisme, en revanche, on dira que la diversité linguistique et la complexité des contextes pour la traduction et la localisation peuvent être gérées par l'innovation que les moyens technologiques disponibles permettent et permettront de mettre en œuvre.

Il est vrai qu'au début du XxI ${ }^{\mathrm{e}}$ siècle la communication dans les langues de moindre diffusion peut s'appuyer sur un outil puissant, les technologies de l'information et surtout la Toile. Les générations du numérique disposent d'un Internet théoriquement multilingue, et certaines langues ont déjà un plus grand volume de contenus écrits en ligne qu'elles n'en ont jamais eu sur papier.

Toutefois, l'instrumentalisation des langues, les ressources en traduction, la localisation de l'environnement logiciel se réalisent pour les très nombreuses langues d'Afrique sub-saharienne à un rythme inégal. La mise en réseau et l'internationalisation des ressources humaines et techniques semblent être un impératif pour les pays de cette région du monde dont la plupart des langues ont depuis longtemps occupé un espace peu valorisant et fait l'objet de stratégies incertaines.

Des obstacles importants sont à gérer: problèmes techniques et économiques d'accès, absence de littéracie, problèmes techniques de disponibilité d'outils d'écriture et de traduction, tensions entre motivations politiques, en particulier entre politique 
«mondialisante» et politiques africaines endogènes; tensions entre motivations individuelles - tendant vers la production de contenus francophones ou anglophones, pour être lu et compris par le plus grand nombre, ou bien vers la localisation et la traduction pour exprimer les identités régionales.

Il y a dans le développement de l'accès et des usages localisés des technologies de l'information en Afrique un enjeu économique qui ne laisse pas la société globale indifférente: en effet, si on veut développer l'économie Internet, il faut créer dans les pays du Sud le potentiel d'interlocuteurs et de partenaires ayant accès à la Toile; or, ceci passe par l'utilisation et l'instrumentalisation des langues locales. On peut dire alors que la mondialisation a besoin de la localisation.

Il n'en demeure pas moins que les acteurs principaux de la localisation sont locaux. L'observation sur le terrain confirme que les pratiques langagières médiatisées par les technologies de l'information, qui impliquent réception ET production de contenus, concernent de plus en plus de personnes, de tous âges et de toutes catégories socioprofessionnelles. Et que si les pays du Nord peuvent partager leur expertise, ce sont les initiatives endogènes, enracinées dans la culture et les besoins locaux, qui pourront y décliner la nécessité de produire des outils de traduction et de localiser les contenus en langues africaines, tout en maintenant la présence des langues de grande diffusion.

Il est important d'être attentif aux comportements des utilisateurs des technologies de l'information, pour passer d'une logique de l'offre à celle d'une réponse adaptée aux demandes, tout en sachant que l'histoire sociopolitique, sociolinguistique et technologique a infléchi celles-ci en direction du recours aux LGC: les habitudes acquises sont à respecter dans les actions de localisation, et il convient de se demander activement à quelles conditions elle est possible et souhaitable, et quelles sont les représentations à faire évoluer pour son déploiement: savoir comment les utilisateurs perçoivent l'existant, quels besoins ils ressentent, est un préalable pour opérer des changements et développer un marché qui soient à la fois conformes à leurs souhaits et dans la logique du principe de respect de la diversité linguistique et culturelle. En effet, c'est au final le contact avec les besoins perçus ou latents qui peut guider les choix à réaliser sur le terrain, et le niveau d'intérêt pour la localisation dans les langues sub-sahariennes sera fonction de l'intérêt des contenus mis à disposition des locuteurs.

On peut alors s'interroger sur les types de contenus ou environnements logiciels à localiser en priorité. Si la Toile qui se tisse est multilingue, si la téléphonie mobile gagne du terrain et si de nouvelles applications sont prévisibles, quel visage la localisation peut-elle donner à la cohabitation entre langues dans l'espace virtuel que constituent l'écran, les liens hypertextes et les nouveaux environnements numériques?

Enfin, il peut être souhaitable que les pays d'Afrique sub-saharienne se construisent un espace d'évaluation de l'utilisation des langues africaines sur les sites institutionnels ou privés, et évaluent aussi les besoins de soutien à la localisation sur la Toile, de manière à déterminer l'incidence du développement de nouvelles ressources localisées sur la préférence jusqu'ici établie vis-à-vis des ressources en LGC, puisque les technologies de l'information sont à la fois outil pour la localisation et vecteur de celle-ci. Et comme partout et plus encore que partout ailleurs, en raison de la fragilité des marchés dans cette région du monde, la problématique de la traduction et de la localisation est liée à des choix politiques. 


\section{NOTES}

1. Dyane Adams a été commissaire aux langues officielles du Canada de 1999 à 2006. Cette citation est extraite d'une conférence plénière qu'elle a prononcée au Congrès international de la FIPF qui s'est tenu à Atlanta en juillet 2004.

2. Observatoire européen du plurilinguisme. Consultée le 19 août 2010, <http://plurilinguisme.europeavenir.com/>.

3. Colloque Traduction des normes et normes de traduction dans l'espace européen: institutions et entreprises. Strasbourg, 23-25 juin 2010. Consultée le 19 août 2010,

$<$ http://www.portalingua.info/fr/actualites/article/traduction-des-normes-et-normes-de-traductiondans-l-espace-europeen/>.

4. Les estimations varient. Selon Lewis (2009), par exemple, il y a au Cameroun 279 langues recensées, alors que les linguistes camerounais ont identifié 239 langues distinctes, une langue étant définie chaque fois comme un ensemble de variantes linguistiques ou de dialectes entre lesquelles l'intercompréhension est satisfaisante.

5. La traduction est aussi un acte politique, nous le savons.

6. E-learning Africa (2006): Localisation, Adaptation et Développement de Contenus. Consultée le 25 mai 2010, <http://www.elearning-africa.com/fra/review_2006_themes.php>.

7. Parler de temps pour les systèmes verbaux des langues africaines est une simplification. Laspect tient une place importante dans ces systèmes.

8. Réseau international francophone d'aménagement linguistique. Consultée le 19 août 2010, <http:// www.rifal.org>.

9. ITU, World Summit on the Information Society. Consultée le 22 mai 2010, <http://www.itu.int/ itu-wsis/implementation/>.

10. L'Institut francophone des nouvelles technologies de l'information et de la formation (INTIF), aujourd'hui Institut de la Francophonie numérique (IFN), organe subsidiaire de l'Organisation Internationale de la Francophonie (OIF), le Fonds Francophone des Inforoutes, le Centre de documentation de l'Agence de la Francophonie (CIFDI), l'Association Internationale des Archives Francophones (AIAF).

11. Création d'un réseau africain consacré à la localisation. Consultée le 10 mai 2010, <http://www. crdi.ca/iicr/ev-117089-201-1-DO_TOPIC.html>.

12. Linguamon Casa de les Llengües. Consultée le 19 août 2010, <http://www10.gencat.cat/casa_llengues/AppJava/fr/casa/Casadelesllengues.jsp >.

13. Institut Panos Afrique de l'Ouest (IPAO). Consultée le 19 août 2010, <http://www.panos-ao.org>.

14. Il fait en particulier mention du colloque tenu à Nairobi en janvier 2007 Diversité culturelle et linguistique: $y$ a-t-il un rôle et un avenir pour les médias en langues nationales? Un enjeu régional pour l'Afrique et un enjeu mondial, où s'est exprimée la tension entre homogénéisation et hétérogénéisation culturelles.

15. A12N Gateway/Portail A12N. Consultée le 19 août 2010, <http://www.bisharat.net/A12N $>$.

16. On peut lire une description du projet sur Afrigen. Consultée le 7 août 2010, <http://www.it46. se/afrigen/?lang $=$ fr $>$.

17. On trouvera un compte-rendu de cette initiative sur WikiAfrica, un outil pour promouvoir une attitude consciente et active de ses utilisateurs. Consultée le 25 mai 2010, <http://www.africultures. com/php/index.php?nav=article\&no=8455>.

18. L'initiative Afrilivres a été lancée par un comité de pilotage d'éditeurs africains réunis en novembre 2001 par Africultures avec l'aide de la Fondation Charles Léopold Mayer pour le progrès de l'homme. Consultée le 10 mai 2010, <http://www.afrilivres.net/index.asp?navig=accueil>.

19. Les rôles et responsabilités des traducteurs en ce qui concerne les normes tant à l'écrit qu'à l'oral avec ces supports restent évidemment entiers.

\section{RÉFÉRENCES}

Aıтo, Emmanuel (2005): L'unité terminologique et les langues en voie de développement: réflexions épistémologiques et ontologiques. Cahiers du RIFAL. 25:6-15.

Ben Henda, Mokhtar (2004): Traitement informatisé des langues, langues nationales et partenariat francophone. Cahiers du RIFAL. 24:61-64.

Conférence régionale sur les Politiques nationales: le rôle des langues transfrontalières et la place des langues de moindre diffusion en Afrique centrale, absence d'auteur (2007): Rapport 
Général. Consultée le 19 août 2010, <http://www.acalan.org/fr/confeven/colloques_ regionaux/rapport_afrique_centrale.pdf $>$.

Diki-KidiRI, Marcel (2004): La recherche sur l'informatisation des langues africaines. Cahiers du RIFAL. 24:65.

Diki-KIdiRI, Marcel (2007a): Éléments de terminologie culturelle. Cahiers du RIFAL. 26:14-25.

DiKI-KIDIRI, Marcel (2007b): Comment assurer la présence d'une langue dans le cyberespace? Consultée le 25 mai 2010, <http://unesdoc.unesco.org/images/0014/001497/149786f.pdf>.

Gaudin, François (2007): Quelques mots sur la socioterminologie. Cahiers du RIFAL. 26:26-35. GuÉDON, Jean-Claude (1996): La planète cyber. L'Internet et le cyberespace. Paris: Gallimard.

Kenmogne, Michel (2002): Traduction Biblique et Mission: Un Défi pour l'Église Africaine. Leçon de clôture, FATEAC de Yaoundé. Consultée le 21 mai 2010, <http://www.cabtal. org/pdf/La_traduction_de_la_bible.pdf $>$.

Lewis, M. Paul, éd. (2009): Ethnologue: Languages of the World, Sixteenth edition. Dallas: SIL International. Consultée le 20 octobre 2010, <http://www.ethnologue.com/>.

Lipou, Antoine (2007): Interventionnisme terminologique et langues en situation minoritaire (avec référence particulière aux langues africaines en zone francophone). Cahiers $d u$ RIFAL. 26:48-57.

Osborn, Don (2010): African Languages in a Digital Age. Challenges and Opportunities for Indigenous Language Computing. Cape Town/Ottawa: HSRC Press/IDRC.

RABENORO, Irène (2005): Les langues des pays du Sud pour l'apprentissage des savoirs du Nord: une illusion? Cahiers du RIFAL. 25:17-28.

Rousseau, Louis-Jean (2007): Élaboration et mise en œuvre des politiques Linguistiques. Cahiers du RIFAL. 26:58-71.

Sommet Mondial sur la Société de l’Information (2005): Agenda de Tunis pour la société de l'information, Document WSIS-05/TUNIS/DOC/6-F. Consultée le 18 mai 2010, <http:// www.itu.int/wsis/docs2/tunis/off/6rev1-fr.html>.

Tervonen, Taina (2003): Traduire le pluriel. Consulté le 18 mai 2010, <http://www.africultures. com/php/index.php?nav=article\&no=2848>.

UNESCO (2003): Recommandation sur la promotion et l'usage du multilinguisme et l'accès universel au cyberespace. Consultée le 22 mai $2010<$ http://portal.unesco.org/ci/fr/ev.php-

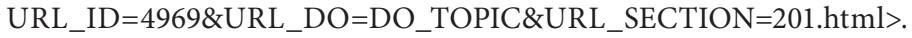

UNESCO (2008a): International Literacy Statistics: a review of concepts, methodology and current data, UNESCO Institute for Statistics, Montreal. Consultée le 22 mai 2010, <http:// www.uis.unesco.org/template/pdf/Literacy/LiteracyReport2008.pdf>.

UNESCO (2008b) : Highlights of UNESCO's involvement in the WSIS follow-up process (2007), Report for the Commission of Science and Technologies for Development, March 2008, Information Society Division.

World Summit on the Information Society (2003): Plan of Action. Document WSIS-03/ GENEVA/DOC/5-E. Consultée le18 août 2010, <http://www.itu.int/wsis/docs/geneva/official/poa.html\#c8>.

YACOB, Daniel (2004): Localize or be localized: An assessment of localization frameworks. Paper presented at the International Symposium on ICT: Education and Application in Developing Countries, (Addis Ababa, Ethiopia 19-21 October).

YANG, Jian (2004): C'est à la technique de s'adapter à l'être humain, pas le contraire. Actes de la vitrine forum sur l'informatisation des langues, Cahiers du RIFAL. 24:68. 